\title{
VIBROfocus: Design of a focal vibro-tactile robotic-assistive system for spasticity rehabilitation
}

\author{
Tijana Jevtic Vojinovic, Aleksandar Zivanovic, Tom Carlson, Rui C.V. Loureiro
}

\begin{abstract}
The main focus of spasticity treatment is to alleviate pain, improve function and reduce risk of additional complications. In this paper the design of a robotic system with enhanced focal vibro-tactile stimulation for the treatment of spasticity in the upper limbs is presented. Building on emerging evidence on the use of vibrations in the treatment of spasticity, we propose a new integrated approach. Our design combines the use of vibro-tactile stimulation of the high tone muscle with robotic movement assistance to augment rehabilitation outcomes in patients with spasticity in the upper limbs.
\end{abstract}

\section{INTRODUCTION}

Advances in rehabilitation are contributing to the reduction of impairment-related morbidity, but in turn are increasing the burden to healthcare systems, as more human and financial resources are needed to deliver therapy. The use of robotic devices, although limited in clinical settings, have the potential to address this problem, as noted by the results of recent studies [1]. When it comes to impairment affecting the upper limbs, further understanding of the underlying recovery mechanisms is needed in order to create and improve assistive robotic devices for every day use in clinical practice and in unsupervised environments. Furthermore the greater understanding of underlying recovery mechanisms is also needed for lower limb impairments.

Spasticity can be characterised as an increased, involuntary, velocity-dependent muscle tone that causes resistance to movement. The condition is often secondary to a disorder or trauma, such as a tumour, a stroke, multiple sclerosis, cerebral palsy or a spinal cord, brain or peripheral nerve injury, and associated with neurodegenerative diseases affecting the upper motor neuron [2]-[4]. It greatly impacts quality of life and activities of daily living, affecting mobility and dexterity, causing pain, deformity and many other repercussions such as limb contracture. Oral medications, which are frequently used for spasticity management, have considerable side effects and limited efficacy [5]. To overcome this problem different externally applied modalities such as vibro-tactile stimulation for

Tijana Jevtic Vojinovic is with School of Science and Technology, Middlesex University London, NW4 4BT, UK and Aspire Centre for Rehabilitation Engineering and Assistive Technology, University College London, Royal National Orthopaedic Hospital, Stanmore, London, HA7 4LP, UK. ((tj172@mdx.ac.uk or t.jevtic (a,ucl.ac.uk)

Aleksandar Zivanovic is with is with School of Science and Technology, Middlesex University London, NW4 4BT, UK. (a.zivanovic@mdx.ac.uk)

Tom Carlson and Rui C. V. Loureiro are with Aspire Centre for Rehabilitation Engineering and Assistive Technology, University College London, Royal National Orthopaedic Hospital, Stanmore, London, HA7 4LP, UK. (t.carlson or r.loureiro@ucl.ac.uk) spasticity reduction have been proposed [6]. Long-term effects of whole body vibrations (WBV) seem to reduce spasticity for up to 6-8 weeks [7]. With limitations of WBV application due to unwieldiness of apparatus, focus needs to be shifted to more easy-to-use technology that can be applied when and where needed (e.g. at home or work place).

This paper proposes an apparatus for focal vibro-tactile stimulation combined with a robotic assistive device to measure and reduce abnormally increased muscle tone and consequent joint stiffness (i.e. spasticity). Following the introduction and background, design considerations are presented in section III and the proposed system in section IV of this paper. A more profound analysis of focal vibro-tactile stimulation is given in the later sections. Section V explains the novel study that includes the application of focal vibro-tactile stimulation to the muscle while recording brain responses. The discussion is presented in section VI followed by the conclusion in section VII.

\section{BACKGROUND}

\section{A. Spasticity rehabilitation}

Spasticity has been described as a tightness experienced with passive movement of the limbs, associated with hypertonicity and/or a combination of positive and negative symptoms of the upper motor neurone syndrome [3]. Spasticity has many definitions [4] typified often as motor disorder that is characterised by a velocity-dependent increase in the tonic stretch reflex (muscle tone). The existence of several definitions, and lack of agreement over which is best, adds to the difficulty in understanding the pathophysiology of spasticity. A more recent definition describes spasticity as "disordered sensory motor control resulting from an upper motor neurone lesion, presenting as intermittent or sustained involuntary activation of muscles" [8].

The main focus of spasticity management is to improve the external impairing causes before treatment is considered. Treatment aims to alleviate pain, improve function, reduce the risk of additional complications, and to assist with maintaining hygiene and transferring [9]. Different treatments are available, ranging from: physical therapy; electrical and functional electrical stimulation (ES/ FES); casting/splinting; and mental imagery to pharmacological interventions and surgery [10].

There is emerging evidence of use of vibrations in treatment of spasticity [11]. Out of three modalities: whole 
body, segmental and focal vibrations, WBV seems to have long lasting reduction on spasticity as well as increase in motor function [12]. However WBV devices are limited to specific and supervised use due to the size and cost of the stimulation delivery device. Nonetheless, more usable vibration devices such as smaller coin shaped motors (referring to segmental or focal vibro-tactile stimulation) have been proposed for spasticity treatment by targeting specific muscle groups [13]-[15]

\section{B. Focal vibrations}

Focal vibro-tactile stimulation appears to have a part to play in preconditioning the healthy muscle to exert greater force [13], [16]. Moreover, Rosenkranz et al showed the corticospinal involvement in muscles followed the transcranial magnetic stimulation (TMS) and the changes in motor evoked potentials [17]. However, Smith showed that these effects are dependent on the timing within the muscle stimulation [18]. That is, changes were only noticeable within the first few seconds of vibro-tactile stimulation onset, and no significant difference after $15 \mathrm{~min}$ from the vibration onset..

Focal vibration frequencies of $100 \mathrm{~Hz}$ applied to triceps muscle are reported to decrease stiffness in elbow and wrist joint in a single case study [11]. Furthermore, prolonged vibration on the proximal leg muscles produced a significant decrease of spasticity in the whole limb despite the differences between complete and incomplete spinal cord injury (SCI) participants [15]. Both of these studies investigated effects of focal vibrations on the participants with SCI, and provide evidence of vibro-tactile stimulation being effective on limbs above and below the level of lesion. With this in mind, the question becomes, "which part of the body (or combination) is responsible for vibro-tactile facilitation: cortical, spinal or local muscular response affects spasticity?"

There are several approaches leading to a better understanding of the correlation between spasticity and vibro-tactile stimulation. This paper proposes two:

- analysis of force, electromyography (EMG) and electroencephalography (EEG)

- analysis of movement parameters and possible neuroplasticity due to movement repetition (e.g. playing a game)

All of these investigations are non-invasive and easy to use in a clinical/research environment. The analysis of the neuro-physiological signals can contribute to adequate selection of vibration parameters, as well as better understanding cortical and muscular responses to stimulation.

Movement analysis can give an insight into the joint stiffness state and muscle tone before and after the intervention [19]. Clinically accepted assessment tools for spasticity, such as the Modified Ashworth Scale (MAS), completely rely on the assessor's subjective feeling of resistance in the limb, therefore a more objective measure is needed. One could propose to exploit the dual nature of robotic assistive devices, to both assist and objectively measure spastic movement. This paper proposes to measure active and passive range of motions with resistance from the limb as an objective measurement of joint stiffness. Combining these measurements with EMG where appropriate, there is a potential for more objective estimation of muscle tone by assessing correlation between EMG from the abnormally increased muscle tone and resistance during range of motion measurements.

\section{DESIGN CONSIDERATIONS}

The spastic arm tends to curl up in an all arm flexion as the spasticity level increases. Volitional movements are almost unmanageable. Often the joint extension of the spastic arm demands the use of external forces (e.g. splints or therapist's help) that needs to be applied carefully, precisely and with minimal pain [20]. The design of a device for the spastic arm/hand rehabilitation needs to take into consideration the ease of positioning the curled arm/hand into the device.

Consultation with clinical colleagues at the Royal National Orthopaedic Hospital, Stanmore, UK, indicated the need for a rehabilitation system that would lower the stiffness in the wrist joint. In clinical practice, treatment first targets stiffness reduction followed by mobilization of the limb and application of FES to stimulate muscles (e.g. grip retraining). A robot-aided spasticity system should therefore consider also the rehabilitation of any residual volitional movements [21].

To ensure safety and comfort, robotic systems need to be easily detached from the user. It is important for the user to be able to see the hand in a system, to balance the ergonomics of the movement and enhance user's comfort. An emergency stop button should always be at hand for the immediate system shut down.

\section{SYSTEM DESIGN}

The VIBROfocus system integrates several elements: a vibration generator, a mechanical wrist robotic manipulandum and a game (please see Fig. 1).

The participant is seated comfortably in a chair facing a computer screen and the robotic manipulandum. The vibrations are applied to the flexors muscles of the forearm. After the stimulation, the hand is positioned in the robotic manipulandum in midsuppination position. The active and passive range of motion of the wrist can be measured and potentially associated with spasticity and to adapt the positions to suit assisted movements requirements of the game.

The software can manage the collection and the control of the data between the wrist device and the game. The level of control over the game is directly proportional to the user's ability to move the wrist device in any of the two directions. 


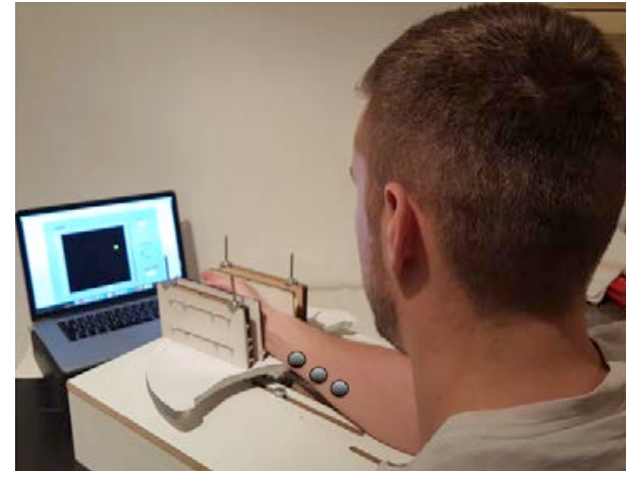

Figure 1. VIBROfocus system. Focal vibro-tactile motors are positioned on the wrist flexors of the forearm. The user is playing a Pong game using robotic manipulandum with assistance to execute movement.

\section{A. Focal vibro-tactile stimulation}

Focal vibro-tactile stimulation will be applied on the flexor muscles of the forearm. The coin shaped vibration motors (Precision MicroDrives ${ }^{\mathrm{TM}}$ ) can be embedded into the flexible velcro strap or a splint to allow easy fixation to the targeted muscles. Recommended parameters for vibration stimulation that will be considered will range between $80-100 \mathrm{~Hz}$ with the amplitude ranging between $0.3-2 \mathrm{~g}$ or more, where $\mathrm{g}$ is a gravity acceleration [11]. The parameters can be easily adjusted depending on the response as they are being controlled by a microcontroller.

\section{B. Mechanical design}

The wrist robotic-aided device comprises of several static and dynamic elements (please see Fig. 2) and taking into account the design considerations presented in the section III.

The elbow is comfortably positioned in a rest splint that can be adjusted depending on the length of the forearm. The hand lies comfortably between two plates in midsuppination position so that the wrist is free to produce flexion and extension. The movement of the hand exerts force on the plates that can be measured by a total of 8 force sensors, embedded behind both contact plates (within support plates).

The hand is fixed between the contact and support plates and on the capstan that can move. The ulnar side of the wrist (ulnar carpal bones) is aligned with the center of rotation of capstan. The capstan movement is achieved by backdrivable motor with encoder (Digilent, 6V, 150RPM) positioned tangential to a half-circled hand rest called capstan. The capstan is driven by a cable wrapped around the motor and the output drums in a figure-of-eight pattern, This is the primary component for power transmission, which is widely used in a capstan driven haptic devices [22].

The capstan center of rotation is connected to the potentiometer via a shaft that measures rotation and is used to control the capstan's position. The capstan has physical limits at both ends to ensure the user's safety, in accordance with healthy wrist range of motion (approx. $180^{\circ}$ ).

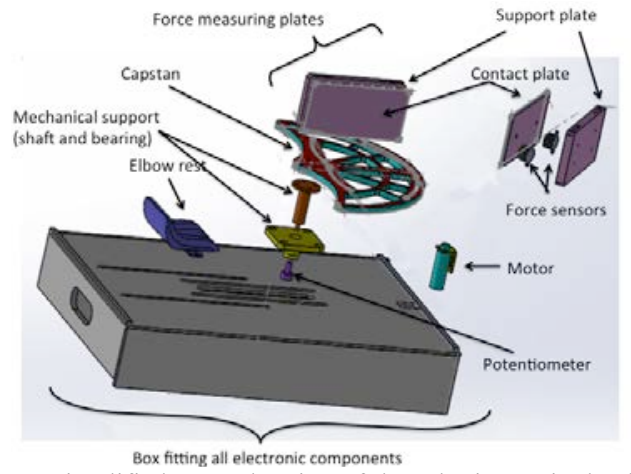

Figure 2. Simplified CAD drawing of the robotic manipulandum. The hand rests between two force measuring plates both equipped with 4 force sensors. The plates and the hand rest on the capstan that is being driven by the cable attached to the motor. The transmission of the capstan rotation is transferred to a potentiometer to measure position.

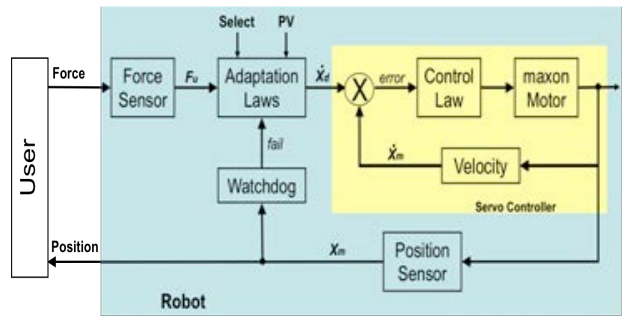

Figure 3. The generalized low-level controller diagram. The controller consists of an outer control loop (darker area) and an inner servo loop (lighter area). A velocity set point vector is generated by the outer loop (adaptation laws) based on position/velocity. The velocity set point value is then used to control the grasp robot in the inner servo loop.

\section{The game}

Patient therapy compliance and motivation is mediated using a gamification paradigm. The well-known "Pong" game has been selected due to its simple, one-player

paradigm and control is achieved with a single degree of freedom (e.g. wrist flexion/extension). The aim of the Pong game is to hit a ball with a paddle, where the paddle is controlled by the user. One of the benefits of the Pong game is that it can be adapted to a multiplayer interface to encourage social rehabilitation.

The Pong game used in VIBROfocus is implemented in LabVIEW, National Instruments ${ }^{\mathrm{TM}}$ (see Fig. 1). It can control and adapt the position and speed of the paddle. The paddle is directly dependent on the patient's wrist movements, which are captured by the change in the capstan's position, as measured by the motor's encoder and potentiometer. This program allows the game dynamics and level of difficulty to be adjusted (e.g. by setting the speed of the ball) depending on the user's needs.

At the beginning of each exercise, the user's range of motion is measured. These measurements are associated with end points of the wrist movements i.e. end positions of the paddle. This is essential in order to assure user's safety.

\section{Control system}

The system was also programed and integrated in LabVIEW (National Instruments ${ }^{\mathrm{TM}}$ ), while data acquisition 
from the sensors (encoder, force sensors and potentiometer) is achieved with the myRIO interface (National Instruments $^{\mathrm{TM}}$ )

The LabVIEW graphical user interface provides the patient or therapist with the ability to easily set up parameters, such as wrist range of motion, game difficulty level, time to complete the game, level of robot assistance

The first mode of the system is the measurement of active range of motion of the wrist. The user is asked to perform maximal flexion and extension of the wrist. The second mode is to measure passive range of motion by driving the wrist to full flexion/extension.

The game can be played with/without assistance. The assistance controller was adapted from the Gentle/G Grasp assistance robot [23]. Fig. 3 shows a simplified diagram of the low-level control loop. The force measured from the user is used to generate an angular velocity set point based on commanded position or velocity. This velocity is fed to the motor, which moves the capstan mechanism (i.e. wrist joint) using a conventional control law. An advantage of this configuration is the ability of the inner loop to cancel the friction and inherited mass of the mechanism. A watchdog monitors acquisition failures, power and encoder failures, thus setting the fail flag that cuts the power to the motors.

\section{FOCAL VIBRO-TACTILE STIMULATION CONSIDERATIONS}

To properly calibrate the focal vibro-tactile stimulation parameters to be used in VIBROfocus system, an EEG study was conducted to analyse the cortical effects of vibration frequency of $80 \mathrm{~Hz}$ and amplitude of $0.3 \mathrm{~g}$ [15]. The analysis presented here discusses the appearance of mu $(\mu)$ waves during the relaxation period, during which focal vibro-tactile stimulation is applied.

The mu waves (approx. range $8-12 \mathrm{~Hz}$ ) that can be observed over sensorimotor cortex are associated with muscle and joint perception and motion coordination. An increase in the mu rhythms can be noticed when the body is relaxed with no intention for movement. $\mathrm{Mu}$ desynchronization causes a decrease in mu power, which occurs when the movement is planned and/or executed [24].

\section{A. EEG experiment method}

Nine able bodied volunteers participated in the experiment (5 males and 4 female aged 18-54). The experiment was performed with approval of Middlesex University Research Ethics Committee (reference number 0764). All the participants gave informed consent to the experimental procedure as required by the Helsinki declaration (1964).

The experimental setup was adapted from a previous study [13] (presented in Fig. 4, left). The participant was asked to rest both hands on a table, in a midsuppination position. To achieve muscle contraction the participant was instructed to abduct the index finger (for either the dominant or non-dominant hand) by pushing against a force transducer. The vibrations were applied over the muscle belly of the first
TABLE I. CONDITIONS OF VIBRATIONS AND CONTRACTION EXECUTIOS

\begin{tabular}{|c|c|c|}
\hline \multirow{2}{*}{ Code } & \multicolumn{2}{|c|}{ Conditions } \\
\cline { 2 - 3 } & Vibrations & $\begin{array}{c}\text { Maximal Voluntary } \\
\text { Contraction (MVC) }\end{array}$ \\
\hline $\mathrm{xD}$ & Not applied & Dominant hand \\
\hline $\mathrm{xN}$ & Not applied & Non dominant hand \\
\hline $\mathrm{NN}$ & Non dominant hand & Non dominant hand \\
\hline $\mathrm{ND}$ & Non dominant hand & Dominant hand \\
\hline $\mathrm{DD}$ & Dominant hand & Dominant hand \\
\hline $\mathrm{DN}$ & Dominant hand & Non dominant hand \\
\hline
\end{tabular}

${ }^{a}$ Code represent abbreviation to represent the condition in further text or figures

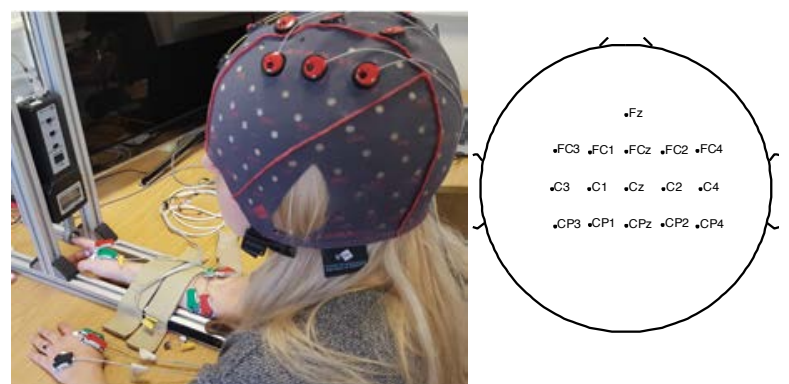

Figure 4. (Left) Experimental set up and (right) the electrode placement on the cap.

interosseous muscle during the relaxation period (i.e. before the contraction). Participants were instructed to 1) keep their eyes closed for the duration of the experiment, 2) to relax entire body when vibro-tactile stimulation was ongoing, 3) to exert maximal voluntary contraction (MVC) against the force gauge with index finger as soon as the vibrations stop. The experiment began with focal vibro-tactile stimulation lasting $30 \mathrm{~s}$ followed by $4 \mathrm{~s}$ of MVC. Each participant engaged in 10 continuous repetitions of this protocol. Both the dominant and non dominant hands were considered in 6 different conditions, as presented in Table I.

Focal vibrations were applied using a small vibration motor $\left(8 \mathrm{~mm}-2 \mathrm{~mm}\right.$ type, Precision MicroDrivers $\left.{ }^{\mathrm{TM}}\right)$ with the frequency generated by the motor modulated to $80 \mathrm{~Hz}$ and amplitude $0.3 \mathrm{~g}$. EEG was recorded using the g.Tec active electorde system (g.GAMMA), with electrodes positioned according to Fig. 4 right, at a sampling frequency of $512 \mathrm{~Hz}$.

EMG was also recorded during the sessions, however the results are not be presented in this paper.

\section{B. Data analysis}

The signal processing and analysis was conducted in MATLAB $^{\circledR}$ using well-established functions and toolboxes. In addition, functions from EEGLab were adapted according to our specific needs [25]. A notch filter was used to cut $50 \mathrm{~Hz}$ (UK mains) from the raw EEG signals. Furthermore, the signals were band-pass filtered between 1 and $40 \mathrm{~Hz}$ using two-way least-square FIR filtering. The adapted surface Laplacian spatial filter was applied using the CSD toolbox [26]. The EEG data was cut into epochs, marked by the borders of vibration onset and offset. The power spectral density (PSD) of each epoch was calculated using the Welch 
method for the period from 0 to $500 \mathrm{~ms}$ in respect to the vibrations onset, $500 \mathrm{~ms}-1 \mathrm{~s}$ the same timings for the end of vibrations. Additionally, each epoch was filtered between 8-12 $\mathrm{Hz}$ to extract mu activity for further analysis, as indicated by our previous study [27].

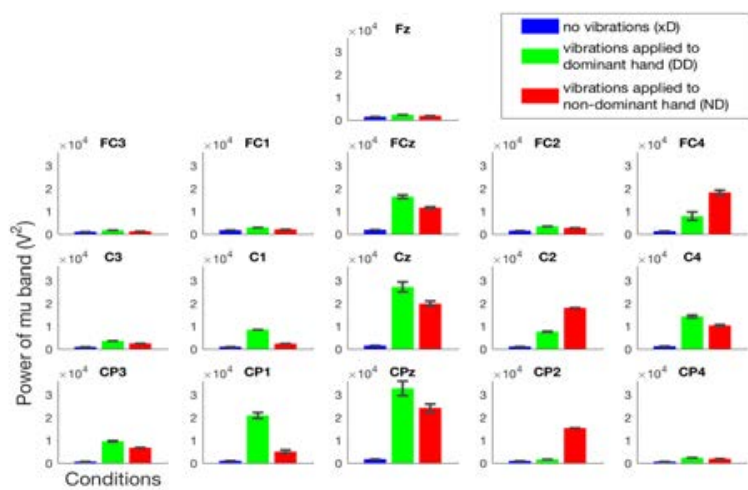

Figure 5-An analysis of mu band power for a single subject. Channels are positioned according to the montage (position on the head). When vibrations are applied, an increase in mu power is noticeable over the contralateral side of the somatosensory cortex (c.f. CP1 versus CP2).

\section{Results}

Repeated measures ANOVA showed no significant difference $(p>0.05)$ in $\mathrm{mu}$ power between conditions where the vibrations are applied on the same side and the change is in the MVC side (conditions DD versus DN and condition NN versus ND from Table I). Therefore the analysis of the results will be focus on the contractions executed by the dominant hand with vibrations not applied (c.f. Table I) vs. applied on the dominant hand (DD) vs. applied on the non-dominant hand (ND). Analysis of the spectrograms across the subject population suggests an increase in mu activity lasting a few seconds from the beginning of the stimulation. This trend then subsides and reappears near the end of the stimulation.

Given that EEG can vary significantly between subjects, Fig. 5 illustrates the observed phenomena for a typical subject. Power of a mu band is represented on Fig. 5 in different colour bars for three different conditions as summarized in Table I: (dominant right hand is contracting) vibrations are not applied (blue), applied to the same hand (green) and applied to non-dominant hand (red). The statistically significant elevation $(\mathrm{p}<0.01)$ in $\mathrm{mu}$ band power is noticeable over the sensorimotor cortex when vibrations are applied, but seems dependent on the side of application. If the vibrations are applied to the dominant hand, the mu band power increases over the contralateral side of the brain (i.e. electrode CP1). The same pattern applies to the vibrated non-dominant hand. In addition, a statistically significant rise in $\mathrm{mu}$ band power is observed over the electrodes $\mathrm{C} 2$ $(\mathrm{p}<0.01)$ and $\mathrm{C} 1(\mathrm{p}<0.05)$.

\section{DISCUSSION}

Combining conventional rehabilitation therapies with robotic devices and adding an automatic monitoring of rehabilitation outcomes could lead to the development of hybrid rehabilitation devices to be used in both clinical and unsupervised (e.g. home) environments. The integration of hybrid robotic devices within intelligent environments could create a future where disability can easily be overcome.

Based on the current literature, it is clear that the even after for example the spasticity level has been reduced, functional manipulation of objects is still limited. Therefore, we hypothesize that if interventional therapies can be used on a daily basis with several assimilated assistive treatments, it can result in better rehabilitation outcomes.

To test this hypothesis we have designed the VIBROfocus system that will be evaluated in a clinical environment with results leading to further variations for multi-center phase II clinical trials. The design takes into consideration several aspects of rehabilitation: 1) relieving treatment - focal vibro-tactile stimulation; 2) movement support - robotic assistive device; 3 ) user engagement - Pong game; and 4) monitoring - EEG, EMG, movement kinematics and level of assistance. The proposed design will be also evaluated for use as a tool for objective diagnosis of joint stiffness and muscle tone reflected in spasticity.A gamification approach is used to deliver motivational therapy with a robotic manipulandum in conjunction with a hybrid admittance-impedance controller to facilitate movement assistance. The robotic manipulandum assists with flexion and extension of the wrist with the motor positioned tangential to a half-circled hand rest. The hand rests between the force sensors to capitalize on the advantages of admittance control. Both the mechanical design and the control strategies minimize system drive backlash and mechanical inertia while executing movements.

A simple robotic manipulandum system is proposed for a single joint. This initial work will allow for a better understanding of how focal vibro-tactile stimulation can be used to alleviate symptoms of spasticity and diagnose changes within. In order to develop a multi-joint focal vibro-tactile robotic-aided system, optimal location/stimulation parameters and integrated sensing system to detect changes in spasticity level must first be devised. As the sensing will be tested within a clinical study, initial parameterization of the stimulation was determined with able-bodied volunteers.

\section{A. Focal vibrations EEG experiment}

Our previous pilot study showed the increase of mu activity over the sensorimotor cortex during the relaxation period during which vibro-tactile stimulation is applied [28]. The results from this study endorse these findings, in that the noticeable increase in mu activity over sensorimotor cortex (see Fig 5, electrodes CP1 and CP2) suggests that the body is relaxed with no intention to move. It can be argued that this contribution is due to the experimental set up, i.e. participants had their eyes closed. Conversely the gain in mu power noticed across $\mathrm{C} 1$ and $\mathrm{C} 2$ electrodes can only be justified with no intention to move during the focal vibration stimulation. Perhaps there is no cortical involvement in the 
response to vibro-tactile stimulation. While recent evidence points towards the use of focal vibro-tactile stimulation to precondition the muscle to increase its force, there is still the question as to which mechanism is responsible for this effect [13], [29].

Kossev et al observed an increase in corticospinal excitability a few seconds after the muscle vibration onset for transcranial magnetic stimulation (TMS) but not for transcranial electrical stimulation (TES) [30]. This leads to the conclusion that there may be a degree of cortical involvement. Smith showed that $15 \mathrm{~min}$ after the onset of muscle vibrations, cortical excitability is unchanged in comparison to the onset of vibrations [18]. We hypothesize that there is cortical involvement in perception of onset of vibrations with transfer of the controlled response to the spinal reflexes. If so, then the vibro-tactile stimulation activates type Ia and $\mathrm{Ib}$ sensory afferents, which react by elevating their firing threshold in order to optimize cortical attention to continue the stimulation. Here, we hypothesize that muscle, spine and brain have a differential effect in excitability due to muscle vibro-tactile stimulation, and that the reaction to stimulation is dependent on the vibration stimulation time [17].

Testing this premise within a population with motor complete and incomplete spinal cord injury could reveal the level of communication and likely organization within focal vibrations to brain to spine to muscle to spastic response. This may not only lead to a more comprehensive understanding of the spasticity mechanisms but also provide insight into the communication between healthy central and peripheral nervous systems.

If focal vibrations' differential effect can contribute to the enhancement of muscle output, then this principle might also be used in the rehabilitation of spasticity. The system could firstly reduce the effects of spasticity (e.g. muscle tone, joint stiffness), and then engage in task-oriented assistive to possibly induce neuroplasticity. The monitoring aspect could also inform the therapists and/or users on the outcomes of the therapy. Hybrid systems such as this could lead to more complete rehabilitation, which increases the patient's independence, whilst minimizing the debilitating aspect of spasticity. Having in mind the differential effects of central nervous system involvement in focal vibration stimulation, we wish to enhance them, to induce neuroplasticity with therapeutic, movement repetition and monitoring aspects of the system.

\section{CONCLUSION AND FUTURE WORK}

This paper presents a hybrid rehabilitation and diagnostics tool for wrist spasticity. The VIBROfocus system includes a focal vibro-tactile stimulation to target spasticity, with a gamification paradigm that assists movements and monitors recovery. We also presented the recommendation of stimulation parameters to be applied to the spastic muscle with justification of the effectiveness.
The VIBROfocus system is to be tested in an ongoing clinical study on both acute and chronic spasticity cases developed after spinal cord injury. The outcome of the clinical study could potentially give an insight into objective measure of spasticity. Furthermore, we aim to better understand the role of spinal involvement in focal vibro-tactile stimulation responses within healthy and spinal cord injury population. Further work in this field could lead to more complete rehabilitation therapies for full recovery including diminishment of the disability repercussions and augmentation of functional movement abilities.

\section{REFERENCES}

[1] P. Maciejasz et al "A survey on robotic devices for upper limb rehabilitation.," $J$ NeuroEngineering Rehabil, vol. 11, no. 1, p. 3, 2014.

[2] S. Malhotra, A. Pandyan, C. Day, P. Jones, and H. Hermens, "Spasticity, an impairment that is poorly defined and poorly measured," Clinical Rehabilitation, vol. 23, no. 7, pp. 651-658, Jun. 2009.

[3] A. Ibuki and J. Bernhardt, "What is spasticity? The discussion continues," Int J Therapy Rehab, vol. 14, no. 9, pp. 391-395, Sep. 2007.

[4] J. W. Lance, Lance: What is spasticity? The Lancet, 1990.

[5] O. Simon and A. P. Yelnik, "Managing spasticity with drugs.," Eur J Phys Rehabil Med, vol. 46, no. 3, pp. 401-410, Sep. 2010.

[6] M. Sadeghi and B. Sawatzky, "Effects of Vibration on Spasticity in Individuals with Spinal Cord Injury," Am J of Phys Medicine \& Rehabilitation, vol. 93, no. 11, pp. 995-1007, Nov. 2014.

[7] M. Sadeghi and B. Sawatzky, "Effects of vibration on spasticity in individuals with spinal cord injury: a scoping systematic review.," Am J Phys Med Rehabil, vol. 93, no. 11, pp. 995-1007, Nov. 2014.

[8] J. H. Burridge,et al "Theoretical and methodological considerations in the measurement of spasticity," Disabil Rehabil, vol. 27, no. 1, pp. 69-80, Jul. 2009.

[9] A. Ward, "Long-term modification of spasticity," Journal of Rehabilitation A. Ward, "Long-term
Medicine-Supplements, 2003.

[10] J. Hsieh, D. L. Wolfe, A. F. Townson, and C. Short, "Spasticity following spinal cord injury," Spinal cord injury ..., 2012

[11] M. Etoom and A. Marchetti, "effect of a focal muscle vibration above triceps brachii muscle on upper limb spasticity in a patient with a chronic spinal cord injury: a case report," IJPR, vol. 3 , no. 4, pp. 1171-1174,. 2015.

[12] M. Y. C. Pang, R. W. K. Lau, and S. P. Yip, "The effects of whole-body vibration therapy on bone turnover, muscle strength, motor function, and spasticity in chronic stroke: a randomized controlled trial.," Eur J Phys Rehabil Med, vol. 49, no. 4, pp. 439-450, Aug. 2013.

[13] T. Jevtic, A. Zivanovic, and R. C. V. Loureiro, "Focal vibro-tactile stimulation as a pre-conditioner to enhance muscle performance in robot-mediated neurorehabilitation," ICORR, 2015, pp. 696-701.

[14] R. Casale,et al "Localized $100 \mathrm{~Hz}$ vibration improves function and reduces upper limb spasticity: a double-blind controlled study.," Eur J Phys Rehabil Med, vol. 50, no. 5, pp. 495504 , Oct. 2014.

[15] H. G. Seo, B.-M. Oh, J.-H. Leigh, C. Chun, C. Park, and C. H. Kim, "Effect of Focal Muscle Vibration on Calf Muscle Spasticity: A Proof-of-Concept Study," $P M \& R$, vol. 8, no. 11, pp. 1083-1089, Nov. 2016

[16] B. Poston, W. R. Holcomb, M. A. Guadagnoli, and L. L. Linn, "The acute effects of mechanical vibration on power output in the bench press.," J Strength Cond Res, vol. 21, no. 1, pp. 199-203, Feb. 2007

[17] K. Rosenkranz and J. C. Rothwell, "Differential effect of muscle vibration on intracortical inhibitory circuits in humans," The Journal of Physiology, vol. 551, no. 2, pp. 649-660, Jul. 2004.

[18] L. Smith, The Effects of Forearm Muscle Vibration on Corticospinal Excitability. 2004.

[19] P. M. Fitts, "The information capacity of the human motor system in controlling the amplitude of movement.," J Exp Psychol, vol. 47, no. 6, pp. 381-391,. 1954.

[20] A. Mukherjee and A. Chakravarty, "Spasticity Mechanisms - for the Clinician," Front. Neur., vol. 1, pp. 1-10, 2010.

[21] R. C. V. Loureiro, W. S. Harwin, R. Lamperd, and C. Collin, "Evaluation of Reach and Grasp Robot-Assisted Therapy Suggests Similar Functional Recovery Patterns on Proximal and Distal Arm Segments in Sub-Acute Hemiplegia," NSRE, IEEE, vol. 22, no. 3, pp. 593-602, 2014.

[22] O. Baser and E. Ilhan Konukseven, "Theoretical and experimental determination of capstan drive slip error," Mechanism and Machine Theory, vol. 45, no. 6, pp. 815-827, 2010.

[23] R. C. V. Loureiro and W. S. Harwin, "Reach \& Grasp Therapy: Design and Control of a 9-DOF Robotic Neuro-rehabilitation System," ICORR 2007., pp. 757-763.

[24] J. A. Pineda, Mirror Neuron Systems. Springer Science \& Business Media, 2009.

[25] A. Delorme and S. Makeig, "EEGLAB: an open source toolbox for analysis of single-trial EEG dynamics including independent component analysis.," Journal of Neuroscience Methods, vol. 134, no. 1, pp. 9-21, Mar. 2004.

[26] J. Kayser and C. E. Tenke, "Principal components analysis of Laplacian waveforms as a generic method for identifying ERP generator patterns: I. Evaluation with auditory oddball tasks," Clinical Neurophysiology, vol. 117, no. 2, pp. 348-368, Feb. 2006.

[27] T. Jevtic, A. Zivanovic, and R. Loureiro, "Cortical and Muscle Response to Focal Vibro-Tactile Stimuli," Converging Clinical and Engineering ..., 2017.

[28] T. Jevtic and A. Zivanovic, "Brain Response to Focal Vibro-Tactile Stimulation Prior to Muscle Contraction," Intelligent Environments pp. 182-185, 2016.

[29] I. Aprile, et al ,"Muscle focal vibration in healthy subjects: evaluation of the effects on upper limb motor performance measured using a robotic device," Eur J Appl Physiol, vol. 116, no. 4, pp. 729-737, Jan. 2016.

[30] A. Kossev, S. Siggelkow, M. Schubert, K. Wohlfarth, and R. Dengler, "Muscle vibration: Different effects on transcranial magnetic and electrical stimulation," Muscle \& Nerve, vol. 22, no. 7, pp. 946-948, Jul. 1999. 